\title{
Digital gangrene as the initial presentation of systemic lupus erythematosus
}

\author{
Olufemi Adelowo, ${ }^{1}$ Hakeem Olaosebikan, ${ }^{1}$ Wahab Ajani, ${ }^{2}$ D T Omosebi ${ }^{2}$ \\ 1Department of Medicine, Lagos State University Teaching Hospital, Lagos, Nigeria \\ ${ }^{2}$ Department of Surgery, Lagos State University Teaching Hospital, Lagos, Nigeria \\ Correspondence to Olufemi Adelowo, femiadelowo2003@yahoo.com
}

\section{Summary}

Systemic lupus erythematosus (SLE) has rarely been reported among African blacks. However, recent reports may indicate otherwise. Digital gangrene in lupus patients has been reported in different populations and has mostly been seen in patients with long-standing lupus. Digital gangrene as the initial presentation of SLE has rarely been reported, even among African blacks. Two cases of digital gangrene as the initial presentation in Nigerian lupus patients are hereby presented. The medical and surgical managements, as well as relevant literature are hereby discussed.

\section{BACKGROUND}

Systemic lupus erythematosus (SLE) is a multisystemic, autoimmune disease with protean manifestations. Previous reports have indicated its rarity in African blacks. ${ }^{1}{ }^{2}$ Symmons ${ }^{3}$ has even proposed a gradient theory to support this. However, recent reports ${ }^{45}$ and our experience have shown that SLE may not be rare.

Digital gangrene coexisting with SLE is uncommonly reported. The earliest reports of this association were by Dubois and Arteberry ${ }^{6}$ and Alarcon-Segovia and Osmundson. ${ }^{7}$ The former found a cumulative incidence of digital gangrene in SLE to be $1.3 \%$. Asherson et al had reported only 27 cases of this association as of 1996. Eighteen cases of lupus digital gangrene had also been reported among the Chinese population as of $2009,{ }^{9}$ while 20 cases were reported among a cohort of 344 Indian SLE patients. ${ }^{10}$ There have been few reported cases of digital gangrene among the black African population. For instance, only one black African was reported in a series of 21 SLE cases from several countries. ${ }^{8}$ Two cases have also been reported from South Africa. ${ }^{11}$

Most cases of lupus with digital gangrene were, however, seen at late stages of the disease. There have been fewer reports of digital gangrene as initial manifestation of SLE. Rosato et al ${ }^{12}$ had even asserted that digital ulcers and gangrene are never present as initial manifestation in SLE. However, Yang et $a l^{13}$ had reported a case of acute gangrene of finger in an 8-year-old Korean girl without prior features of SLE. Cheah ${ }^{14}$ had also reported a 33-year-old Chinese woman presenting with digital gangrene 3 weeks after the diagnosis of SLE. Other case reports have been documented from UK. ${ }^{15}{ }^{16}$ There has, however, been no documented report of digital gangrene as the initial manifestation of SLE among African blacks.

We hereby present two female Nigerians with SLE who presented initially as digital gangrene.

\section{CASE PRESENTATION \\ Case 1}

$\mathrm{CO}$ is a 27-year-old Nigerian woman who presented to the medical outpatient clinic of Lagos State University Teaching Hospital, Ikeja (LASUTH) with a preceding 2 -month history of digital gangrene and ulcers. These involved the left big toe, second toe as well as the right second and third digits. The gangrene was preceded by blisters and pain in the toes. She also gave a recent history of recurrent fever, polyarthritis, fatigue and weight loss. Polyarthritis involved the wrists, metacarpophalangeal, proximal interphalangeal and knee joints. She denied any preceding trauma and there were no clinical features suggestive of diabetes mellitus and dyslipidaemia. There were also neither symptoms suggestive of lupus or scleroderma nor of Raynaud's phenomenon.

Physical examination showed a young lady who was afebrile, not ill looking but she was pale. There was no significant lymphadenopathy. Examinations of all the systems were essentially normal, except for that of the musculoskeletal system. This revealed gangrene and necrotic ulcers on the toes of both feet, especially the left (figures 1 and 2). The dorsalis pedis and posterior tibial arteries of both feet were, however, palpable and pulsatile.

Laboratory investigations showed a haematocrit of $26 \%$ with normal total white blood cell count and differentials. The erythrocyte sedimentation rate (ESR) was elevated to $120 \mathrm{~mm} / \mathrm{h}$. C reactive protein (CRP) measured $121.4 \mathrm{mg} / \mathrm{l}$ (normal 0-8 mg/l). Serology showed positive antinuclear antibody (ANA) titre 1:640, rim pattern on immunofluorescence. Antidouble-stranded (ds) DNA was positive at $80 \mathrm{IU} / \mathrm{ml}$ (normal $<50 \mathrm{IU} / \mathrm{ml}$ ) while extractable nuclear antigen (ENA) screen was positive. Antineutrophil cytoplasmic antibody (ANCA) was negative while anticardiolipin antibody (ACA) and $\beta 2$ glycoprotein I ( $\beta 2 \mathrm{GPI}$ ) were also negative. Lupus anticoagulant was negative as 


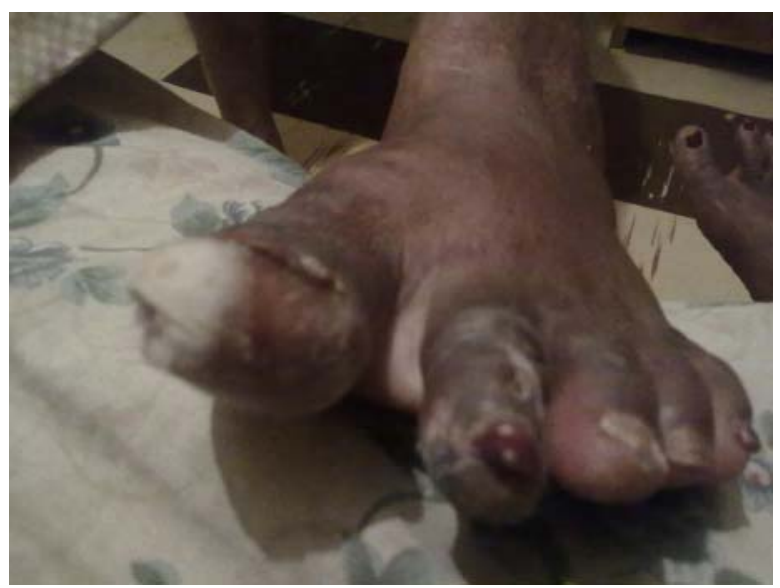

Figure 1 Patient 1 showing gangrene and ulceration of the first two digits of the left foot.

well as serology for HIV, hepatitis B and C. Serum cryoglobulin and cryofibrinogen were both negative. Random blood sugar and lipid profile were within normal limits. Liver and kidney function tests were also within normal limits. Urine dipstick and microscopy did not reveal any abnormalities. Radiographs of the feet did not show any underlying bony pathology. Doppler ultrasound of both lower limbs was reported as normal.

Diagnosis of lupus digital gangrene was made. She was placed on pulse methylprednisolone $1 \mathrm{~g}$ daily for 3 days, as well as pulse cyclophosphamide $500 \mathrm{mg}$ every 2 weeks for 3 months. Administration of pulse methylprednisolone was followed with tablet prednisolone $40 \mathrm{mg}$ daily. Azathioprine tablets $150 \mathrm{mg}$ daily was given in divided doses following the completion of the course of pulse cyclophosphamide. Hydroxychloroquine tablets, $200 \mathrm{mg}$ twice daily was also given. She was comanaged with the plastic surgery unit who performed wound debridement and dressing. She did improve and at the end of 6 weeks, the gangrene had receded and the wound had healed (figure 3). Over the next 9 months, the patient showed improvement in her clinical parameters and laboratory indices with the ESR down to $5 \mathrm{~mm} / \mathrm{h}$.

The patient is still being followed up.

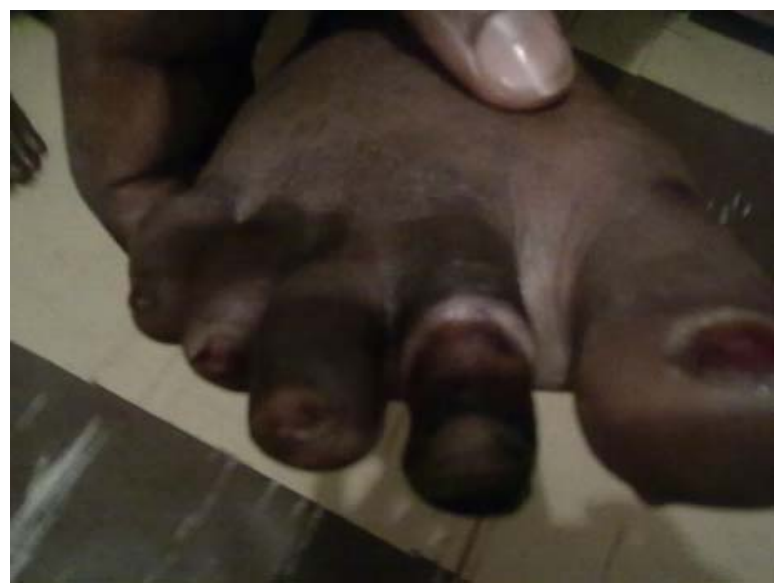

Figure 2 Patient 1 showing digital gangrene of toes of the right foot.

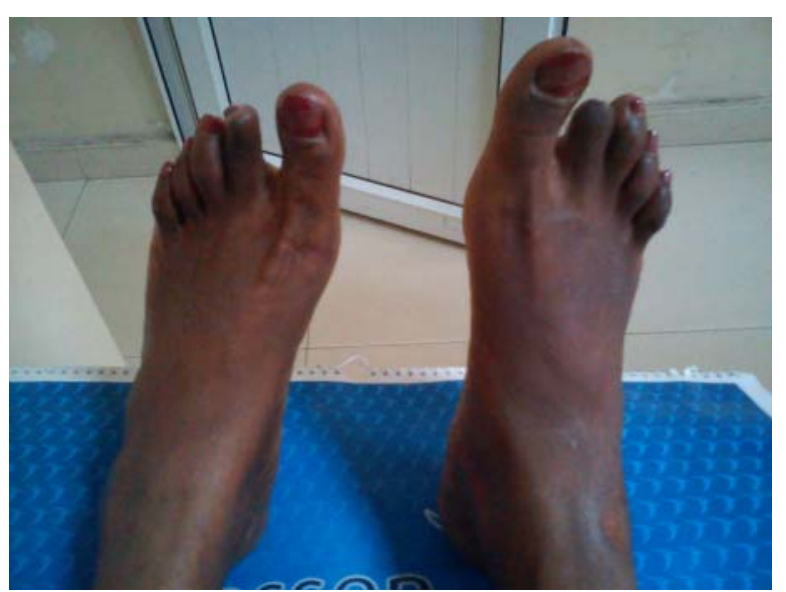

Figure 3 Both feet of patient 1 after treatment and dressing.

\section{Case 2}

$\mathrm{AL}$ is a 32-year-old woman who was initially admitted into the plastic surgery unit and referred to the rheumatology unit. She gave a 3-month history of rapidly progressive bilateral dusky discolouration of the digits of both hands and feet. This had resulted in autoamputation of the second to fifth digits as well as ulceration of the right foot. She also gave a history of Raynaud's phenomenon. She had noticed progressive weight loss, fever and fatigue, as well as polyarthralgia and mouth ulcers within the preceding 1 month but no history of skin rashes, or sicca symptoms. Her haemoglobin genotype is AA.

Physical examination revealed an ill-looking young woman with pallor; and three small lymph nodes in the left cervical region. She was hypertensive with blood pressure of $160 / 100 \mathrm{~mm} \mathrm{Hg}$. All the peripheral vessels were palpable and pulsatile. Examination of all the systems was essentially normal except for the musculoskeletal system. This showed dry gangrene involving the digits of the hands and left foot. There were also autoamputation of the toes of the right foot as well as a necrotic foul-smelling ulcer on the dorsum of the right foot with the tendons visible (figures 4 and 5). Both hands were grossly gangrenous though there were no ulcers (figure 6).

Laboratory investigations-haematocrit $21 \%$ with normal total differential white blood cell count. ESR was elevated to $102 \mathrm{~mm} / \mathrm{h}$. CRP was normal. Radiograph of the feet showed autoamputation of the second to fifth toes of the right foot. Chest radiograph showed cardiomegaly while echocardiography showed marked concentric left ventricular hypertrophy with diastolic dysfunction. Abdominal ultrasound was essentially normal except for the presence of uterine fibroids.

Serology showed positive ANA, titre 1:320, homogeneous staining pattern. Anti-ds DNA was positive at $150 \mathrm{IU} / 1$ so also the ENA screen. Anti-B2GPI (immunoglobulin (Ig) G) was moderately positive at a titre of $47 \mathrm{gpl} / \mathrm{ml}$ (normal 0-20). Lupus anticoagulant and ANCA were, however, negative. Serum cryoglobulin and cryofibrinogen were both negative. Urine dipstick and microscopy did not reveal any abnormalities. Liver and kidney function tests were essentially normal. Serology for HIV, hepatitis $\mathrm{B}$ and $\mathrm{C}$ were also negative. Doppler ultrasound of both legs was reported as normal. 


\section{BMJ Case Reports}

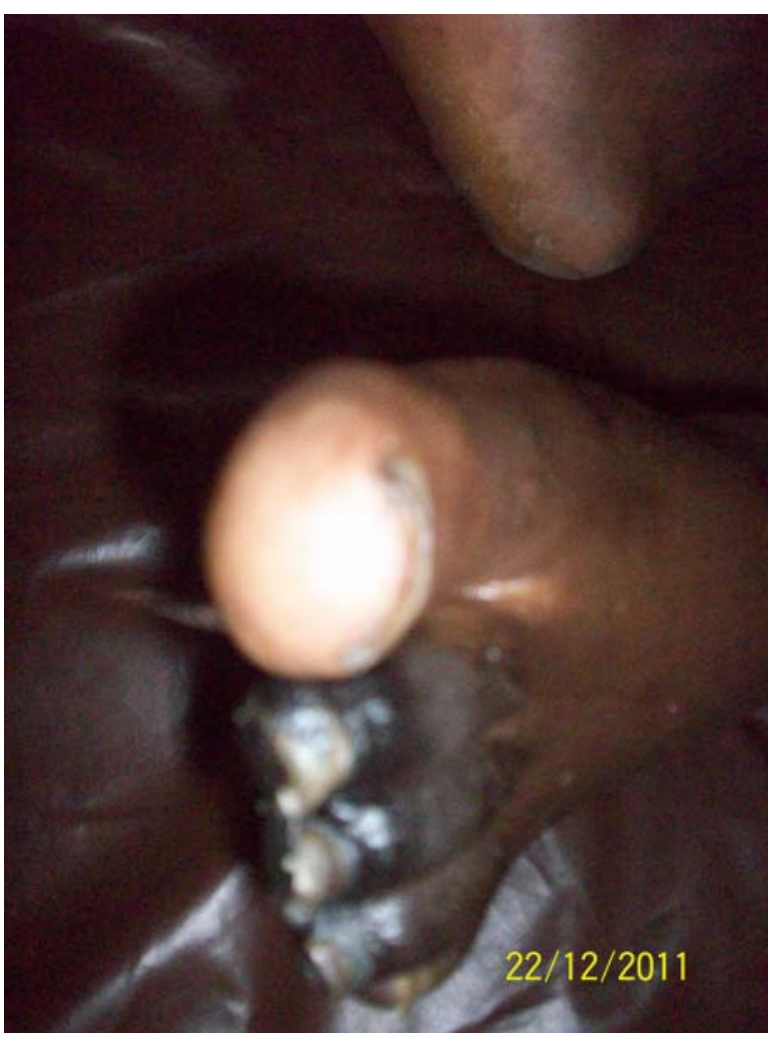

Figure 4 Left foot of patient 2 showing gangrene of the digits.

The diagnosis of lupus digital gangrene with antiphospholipid syndrome (APS) was made.

The patient was treated essentially as in case 1 but with the addition of warfarin and aspirin. The plastic surgery unit performed debridement, wound dressing and eventually disarticulation with scheduled skin graft at a later date. Patient made remarkable improvement and at 6 weeks, there were obvious lines of demarcation of the fingers while the foot ulcer had started to granulate (figures 7 and 8). After 1 year, repeat ESR was normal at $12 \mathrm{~mm} / \mathrm{h}$ while CRP was also within normal limits at less than $50 \mathrm{IU} / \mathrm{ml}$.

The patient is still being followed up.

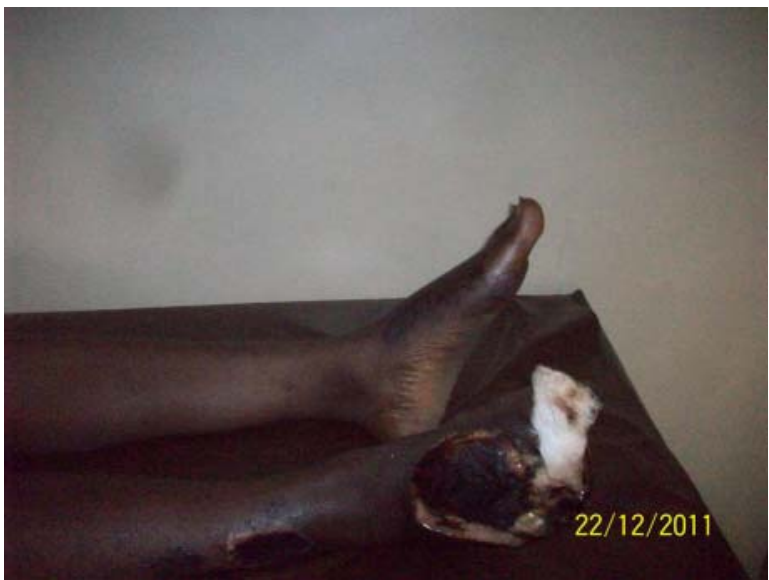

Figure 5 Right foot of patient 2 showing severe gangrene and ulceration.

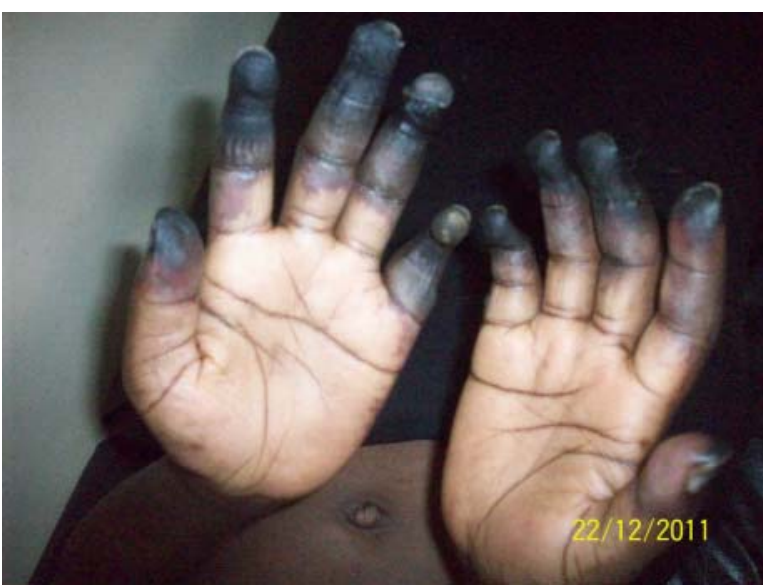

Figure 6 Both hands of patient 2 showing gangrene in the digits of both hands.

\section{INVESTIGATIONS}

Full blood count, ESR, ANAs, double-stranded DNA, ENA screen, ACA, anti-32GPI, ANCA, radiography, Doppler ultrasound of lower limbs and abdominal ultrasound.

\section{DIFFERENTIAL DIAGNOSIS}

Diabetes mellitus is the commonest cause of digital gangrene in our population. This was investigated and excluded. Atherosclerosis is an uncommon cause but this was excluded. There is a remote possibility of ANCA-associated vasculitis but the serology was not supportive of the diagnosis.

\section{TREATMENT}

Both patients were admitted and comanaged with the plastic surgical unit. Antibiotics, corticosteroids, immunosuppressives and anticoagulants were given. Wounds were dressed with debridement being performed. Disarticulation and skin draft were also carried out.

\section{OUTCOME AND FOLLOW-UP}

Patients' wounds healed and they are still being followed up in both the rheumatology and plastic surgery clinics.

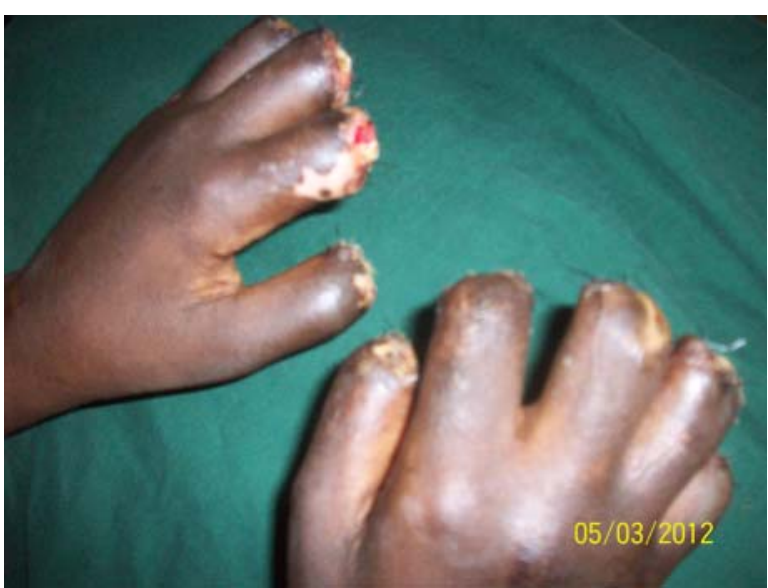

Figure 7 Hands of patient 2 following treatment and surgery. 


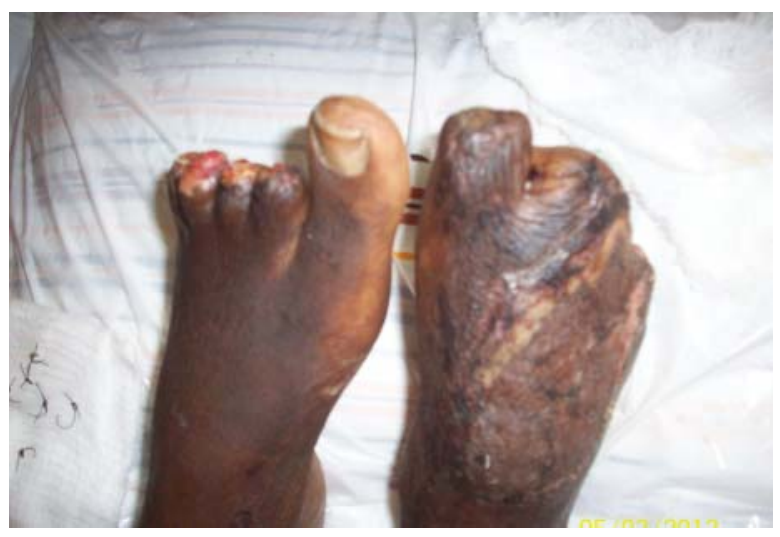

Figure 8 The feet of patient 2 following treatment and surgery.

\section{DISCUSSION}

These two cases are presented to highlight an unusual presentation of SLE, especially among a population hitherto reported to rarely have lupus. Both patients had digital gangrene as their initial presentation in the hospital. Other clinical features and serology confirmed the diagnoses of SLE.

The aetiology of digital gangrene in SLE is complex and may involve several factors including APS, vasculitis, premature atherosclerosis, vasospasm and thromboembolism. Liu et al ${ }^{9}$ have also suggested other contributory factors such as disease duration, Raynaud's phenomenon, elevated CRP and dyslipidaemia. Plasma cholesterol and triglycerides were normal in both patients and only one patient had elevated CRP. ENA screens were, however, positive in both patients, although the constituent analyses were not performed owing to the attendant cost.

APS has been closely associated with lupus digital gangrene; the condition being seen in $3.3-7.5 \%$ of APS. ${ }^{17} 18$ A report from Germany found an increase in ANA and $\beta 2 \mathrm{GPI}$ in a group of patients presenting with peripheral arterial disease. ${ }^{19}$ Galli et al ${ }^{20}$ have suggested that IgG $\beta 2 \mathrm{GPI}$ is an important risk factor for vascular thrombosis, this association being particularly high in SLE. Other reports have described association between antiphospholipid antibodies and ulceration, gangrene and superficial venous thrombosis. ${ }^{182122}$ One of the two patients in our report had elevation of this serological marker which is similar to the single case of positive $32 \mathrm{GPI}$ among the three cases of digital gangrene reported by Nagai et al. ${ }^{23}$ While the association is seen, it appears that the presence of anticardiolipin antibodies is not invariable. Other factors may thus be important as well.

Raynaud's phenomenon has been associated with digital gangrene. In the report by Alarcon-Segovia and Osmundson ${ }^{7}$, three of the seven patients had Raynaud's phenomenon though Dubois and Arteberry ${ }^{6}$ reported only one of five cases presenting with this. One of our patients presented with Raynaud's phenomenon

Early treatment with aggressive corticosteroid, immunosuppressives and lipid-lowering agents and anticoagulation has been suggested. Treatment with corticosteroids decrease the hazard of amputation. ${ }^{9}$ Our patients had pulse methylprednisolone, cyclophosphamide, while the patients with APS also had anticoagulation with warfarin and aspirin. The outcome of the management was very good as indicated in figures 3, 6 and 7 .

These two patients with SLE associated with digital gangrene are presented to heighten the index of suspicion of this unusual presentation this association in black Africans. This is more so in a population hitherto said to rarely have SLE.

Learning points

- Digital gangrene may be seen in black African lupus patients at the onset of the disease.

- Serological markers are important in determining management strategy.

- Corticosteroids and immunosuppressives, among others, are important in its management.

- Collaborative management with plastic surgeons is essential.

\section{Competing interests None.}

Patient consent Obtained.

\section{REFERENCES}

1. Molokhia M, Mekeique PM, Cuadrado M, et al. Systemic lupus erythematosus in migrants from West Africa compared with Afro-Caribbean people in UK. Lancet 2001;357:1414-15.

2. Bae SC, Fraser P, Liang MH. The epidemiology of systemic lupus erythematosus in population of African ancestry. Arthritis Rheum 1998:41:2091-9.

3. Symmons DP. Frequency of lupus in people of African origin. Lupus 1995:4:176-8.

4. Adelowo 00, Oguntona SA. Pattern of systemic lupus erythematosus among Nigerians. Clin Rheumatol 2009;28:699-703.

5. Gilkeson GS, James JA, Kamen DL, et al. The United States to Africa lupus prevalence gradient revisited. Lupus 2011:20:1095-103.

6. Dubois EL, Arteberry JD. Gangrene as a manifestation of systemic lupus erythematosus. J Am Med Assoc 1962;181:366-74.

7. Alarcon-Segovia D, Osmundson PJ. Peripheral vascular syndrome associated with systemic lupus erythematosus. Ann Intern Med 1965;62:907-19.

8. Asherson RA, Cervera R, Shoenfield Y. Peripheral vascular occlusions leading to gangrene in antiphospholipid antibody positive patients. Ann N Y Acad Sci 2007:1108:515-29.

9. Liu A, Zhang W, Tian $X$, et al. Prevalence, risk factors and outcome of digital gangrene in 2684 lupus patients. Lupus 2009;18:1112-18.

10. Rajasekhar L, Jayachandran NV, Prabu VN, et al. Comment on: prevalence, serological features, response to treatment and outcome of critical peripheral ischaemia in a cohort of lupus patients. Rheumatology 2009:48:451-2.

11. Jessop S, Meyers OL. Systemic lupus erythematosus in Cape Town. S Afr Med J 1973;47:222-5.

12. Rosato E, Molinaro I, Pissari S, et al. Digital ulcers as an initial manifestation of systemic lupus erythematosus. Intern Med 2011;50:767-9.

13. Yang SG, Kim KH, Park KC, et al. A case of systemic lupus erythematosus showing acute gangrenous changes of fingertips. $\mathrm{Br} \mathrm{J}$ Dermatol 1996;134:185-7.

14. Cheah JS. Systemic lupus erythematosus in a Chinese woman presenting with gangrene of the fingers. Aust N Z J Med 1973;3:197-9.

15. Jindal BK, Martin MF, Gayner A. Gangrene developing after minor surgery in a patient with undiagnosed systemic lupus erythematosus. Ann Rheum Dis. 1983;42:347-9.

16. Gladstein OS, Rynes Rl, Parhami N, et al. Gangrene of a foot secondary to systemic lupus erythematosus with large vessel vasculitis. J Rheumatol 1979;6:549-53.

17. Knarik G. Clinical characteristics of patients with systemic lupus erythematosus with anticardiolipin antibody. APLAR J Rheumatol 2006; (Suppl 1):A 21-278. 


\section{BMJ Case Reports}

18. Asherson RA, Frances C, laccarino L, et al. The antiphospholipid antibody syndrome: diagnosis, skin manifestations and current therapy. Clin Exp Rheumatol 2006;24:S46-51.

19. Kreger K, Kreuzfelder E. Auto antibodies and peripheral arterial occlusive disease. Herz 2004;20:26-3119.

20. Galli M, Lucini D, Bertolini G, et al. Anti beta 2 glycoprotein I, antiprothrombin antibodies and the risk of thrombosis in the antiphospholipid syndrome. Blood 2003;102:2717-23.
21. Paira S, Roverano S, Zunino A, et al. Extensive cutaneous necrosis associated with anticardiolipin antibodies. J Rheumatol 1999;26:197-200.

22. Vasugi Z, Danda D. Systemic lupus erythematosus with antiphospholipid syndrome: a mimic of Buerger's disease. J Postgrad Med 2006;52:132-3.

23. Nagai $Y$, Shimizu $A$, Suto $M$, et al. Digital gangrene in systemic lupus erythematosus. Acta Derm Venereol 2009;89:398-401.

Copyright 2012 BMJ Publishing Group. All rights reserved. For permission to reuse any of this content visit http://group.bmj.com/group/rights-licensing/permissions.

BMJ Case Report Fellows may re-use this article for personal use and teaching without any further permission.

Please cite this article as follows (you will need to access the article online to obtain the date of publication).

Adelowo 0, Olaosebikan H, Ajani W, Omosebi DT. Digital gangrene as the initial presentation of systemic lupus erythematosus. BMJ Case Reports 2012;10.1136/bcr-2012-006259, Published XXX

Become a Fellow of BMJ Case Reports today and you can:

- Submit as many cases as you like

- Enjoy fast sympathetic peer review and rapid publication of accepted articles

- Access all the published articles

- Re-use any of the published material for personal use and teaching without further permission

For information on Institutional Fellowships contact consortiasales@bmjgroup.com

Visit casereports.bmj.com for more articles like this and to become a Fellow 